Proc. Estonian Acad. Sci. Eng., 2004, 10, 3, 209-226

\title{
Transients in adaptive Fourier analysers
}

\author{
Ants Ronk and Ülle Voolaine \\ Department of Electronics, Tallinn University of Technology, Ehitajate tee 5, 19086 Tallinn, \\ Estonia; ronk@ttu.ee \\ Received 6 April 2004, in revised form 17 June 2004

\begin{abstract}
In the paper several different modifications of an adaptive Fourier analyser are considered presenting simulation results on transients of the analyser's fundamental frequency and output signal. The results permit to compare convergence properties of these modifications and to choose and optimize a proper modification for different applications, including such an extension of the analyser, which processes composite signals consisting of several periodic signals of
\end{abstract} \\ different waveforms and (not harmonically related) frequencies.
}

Key words: adaptive filters, observers, spectral analysis, waveform analysis, frequency estimation, transient processes.

\section{INTRODUCTION}

In the process of development of the analyser for the reproduction and analysis of signal's periodic components of different frequencies and waveforms by means of extension of a block-adaptive Fourier analyser (BAFA), several problems have arisen $\left.{ }^{1,2}\right]$. The most serious one is that in certain cases the output signal's transients (oscillations) appear to be huge compared to the signal. It has been supposed that this difficulty may be overcome by making changes in the structure of the analyser. In this paper, we consider several modifications of an adaptive Fourier analyser (AFA) and give a comparison of their practical convergence properties presenting transients of the analyser's fundamental frequency and signals. These results form a basis for picking out the AFA, which is the most suitable for processing periodic signals with clearly dominating higher harmonic components (so that the level of output signals and the frequency convergence speed are both acceptable). We suppose that extending such AFA one can obtain a better tool for processing composite signals with several periodic components of not harmonically related frequencies. 
In the following we outline basic features of AFAs and describe the performed computer experiments and their objects. Finally the obtained results and conclusions are presented.

\section{PRELIMINARIES}

\subsection{Basic structure of the adaptive Fourier analyser}

The observer-based methods for recursive discrete transformations of periodic signals have been developed by Hostetter $\left[^{3,4}\right]$ and Péceli $\left.{ }^{5}\right]$. The resonator structure proposed in $\left[^{5}\right]$ and shown in Fig. 1 is used in several different adaptive Fourier analysers and it is characterized by the state equations

$$
\begin{aligned}
& \hat{\mathbf{X}}(k+1)=\hat{\mathbf{X}}(k)+\mathbf{g}(k) \cdot[x(k)-\hat{x}(k)], \\
& \hat{x}(k)=\mathbf{c}^{T}(k) \cdot \hat{\mathbf{X}}(k),
\end{aligned}
$$

where

$$
x(k)=\sum_{m=-M}^{M} X_{m}(k) e^{j \omega m k} \quad(j=\sqrt{-1})
$$

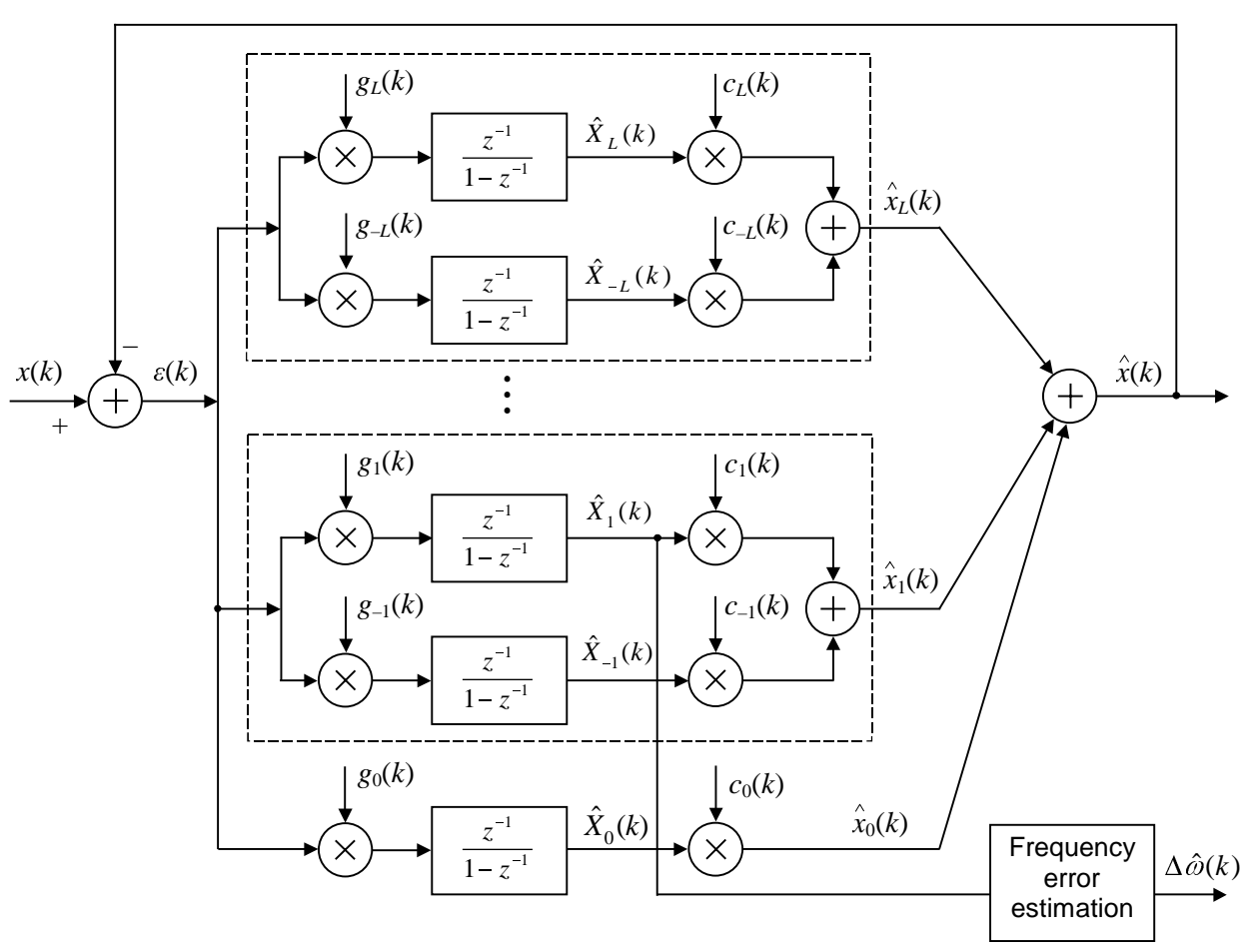

Fig. 1. Block diagram of an adaptive Fourier analyser. 
is the current value (the $k$ th sample) of the assumed band-limited periodic input signal of the angular frequency $\omega ; \hat{x}(k)$ is the estimate of $x(k)$ found by the analyser; $\hat{\mathbf{X}}(k)=\left(\hat{X}_{-L}(k), \hat{X}_{-L+1}(k), \ldots, \hat{X}_{0}(k), \hat{X}_{1}(k), \ldots, \hat{X}_{L}(k)\right)^{T}$ is the statevector and its element $\hat{X}_{l}(k)$ (with the initial value $\left.\hat{X}_{l}(0)=0\right)$ is the estimate of the input signal's complex Fourier coefficient $X_{l}(k),-L \leq l \leq L$, where $L$ is the number of the harmonic component in $\hat{x}(k)$ and $N=2 L+1$ is the number of resonators in the basic observer structure and also the length of the vectors in (1); $\mathbf{c}(k)=\left(c_{-L}(k), c_{-L+1}(k), \ldots, c_{0}(k), c_{1}(k), \ldots, c_{L}(k)\right)^{T}$ is a vector of frequencydependent time-varying coefficients, i.e., signals, which are modulated by corresponding Fourier coefficients in $\mathbf{X}(k)$ to obtain components of the output signal $\hat{x}(k)$. The elements of $\mathbf{c}(k)$ are such that

$$
c_{l}(k)=c_{l}(k-1) z_{l}, \quad z_{l}=e^{j \hat{\omega} l}, \quad c_{l}(0)=1,
$$

where $\hat{\omega}$, the fundamental angular frequency of the analyser (its output signal $\hat{x}(k))$, is an estimate of the input signal's angular frequency $\omega$.

In Eq. (1) the vector $\mathbf{g}(k)$ can be written as

$$
\mathbf{g}(k)=\left(g_{-L}(k), g_{-L+1}(k), \ldots, g_{0}(k), g_{1}(k), \ldots, g_{L}(k)\right)^{T}
$$

and it can be considered as a vector of signals, which are used to demodulate the error signal $\varepsilon(k)=x(k)-\hat{x}(k)$. One can use different frequency-dependent timevarying coefficients

$$
g_{l}(k)=\left(G_{l} c_{l}(k)\right)^{-1} .
$$

In this paper we consider the next two cases:

$$
G_{l}=N
$$

which is used in AFA $\left[{ }^{6}\right]$ and leads to infinite but quite fast decaying transients, and

$$
G_{l}=r_{l}^{-1}=\prod_{\substack{i=-L \\ i \neq l}}^{L}\left(1-z_{i} \cdot z_{l}^{-1}\right),
$$

which is applied in the BAFA $\left[^{7}\right]$ to make the observer a dead-beat one with the length of transients not exceeding $N$ time-steps.

The mentioned output signal's transients appear due to changes in the input signal or as a result of updating $\hat{\omega}$ and also the vectors $\mathbf{c}(k)$ and $\mathbf{g}(k)$. In the updating process, $\hat{\omega}$ obtains a new value

$$
\hat{\omega}_{\text {new }}=\hat{\omega}+\Delta \hat{\omega},
$$

where $\Delta \hat{\omega}$ is the estimate of the error $\Delta \omega=\omega-\hat{\omega}$. The length $N$ of the vectors in (1) changes too as $L$ is always updated so that 


$$
\pi / \hat{\omega}-1 \leq L<\pi / \hat{\omega}
$$

holds. If $N$ increases, then $\hat{\mathbf{X}}(k)$ is appended with zeros and $\mathbf{c}(k)$ with ones.

After setting $\hat{\omega}=\omega$, the transient process tends towards $\hat{x}(k)=x(k)$ and $\hat{X}_{l}(k)=X_{l}(k)$ for $l=-L,-L+1, \ldots, L$ with $L=M$. In case of $\hat{\omega} \neq \omega$ one has to find $\Delta \hat{\omega}$ and to update $\hat{\omega}$ and all the frequency-dependent parameters of the analyser according to Eqs. (2)-(7).

\subsection{Estimation of the frequency error}

The error estimate $\Delta \hat{\omega}$ is found using the fact that the first Fourier coefficient $\hat{X}_{1}(k)$ can be considered as a vector in the complex plane and, when the transients have decayed, this vector rotates in case of $\hat{\omega} \neq \omega$ with an average speed of $\Delta \omega$. Its actual rotation speed is $\Delta \omega+\Delta \omega_{\mathrm{d}}(k)$ where $\Delta \omega_{\mathrm{d}}(k)$ is a sum of periodic disturbances caused by other Fourier components of the signal $x(k)$.

The estimate $\Delta \hat{\omega}$ can be found in different ways.

1. In the AFA $\left[{ }^{6}\right]$

$$
\Delta \hat{\omega}(k)=\angle\left(\hat{X}_{1}(k) / \hat{X}_{1}(k-1)\right) / N(k)
$$

and it is used for frequency updating in every time-step in spite of disturbing infinite transients (here and further $\angle(X)$ denotes the angle of the complex vector $X)$. These transients compensate each other partially and their summary influence is suppressed by means of implicit averaging over $N(k)$ samples in the updating process.

2. In the BAFA $\left[{ }^{7}\right]$, the block-adaptation is applied and the error estimate

$$
\Delta \hat{\omega}=\angle\left(\hat{X}_{1}(k) / \hat{X}_{1}(k-P)\right) / P
$$

is found during $P+1$ time-steps and only after the finite transient processes of length $N$ (caused by previous frequency updating) have ended.

3. The paper $\left[{ }^{8}\right]$ recommends to use instead of (9) a more general and potentially more efficient error estimation

$$
\Delta \hat{\omega}=\angle\left(\hat{X}_{1, B}(k) / \hat{X}_{1, B}(k-P)\right) / P,
$$

where

$$
\hat{X}_{1, B}(k)=\frac{1}{B} \sum_{b=0}^{B-1} \hat{X}_{1}(k-b)
$$

is a conditioned (averaged) estimate of the signal's first Fourier coefficient vector.

The above three approaches use averaging to suppress disturbances and assume that the input signal's waveform and frequency do not change or change slowly enough. Some simple considerations allow us to hope that in case of 
$P=B \approx N$, Eq. (10) is time-optimal and gives up to twice faster frequency convergence than (9), i.e., (10) with $P \approx N, B=1$.

\section{SET-UP OF EXPERIMENTS AND INVESTIGATED ANALYSERS}

In our experiments we used different analysers with zero initial states and with varied values of their parameters to perform analysis of several signals of the frequency $\omega=0.2$. The relative error $\delta_{\omega}=\Delta \omega / \omega$ of the analyser's initial frequency $\hat{\omega}$ was +0.15 or -0.15 . We found the times $t_{1}$ and $t_{2}$, which were needed to achieve the output signal's frequency error levels $\left|\delta_{1}\right|=10^{-7}$ and $\left|\delta_{2}\right|=10^{-14}$, respectively. These times were found for every input signal (and for all the used parameter values, i.e., for the tunings of the analyser) and often averaged over the set of randomly chosen initial phases of the input signal. The normalized times $t_{1} / T$ and $\Delta t / T$, where $T=2 \pi / \omega$ and $\Delta t=t_{2}-t_{1}$ (which is not influenced by large initial frequency error), characterize thus practical and nearly asymptotic convergence of $\hat{\omega}$.

The band-limited discrete-time periodic signals, which were used to estimate capabilities of the analysers, are presented in Fig. 2. These signals are formed according to their Fourier series by means of a generator based on the conceptual model of the signal assumed in the analyser's structure (1). This numerical generator forms its output signal as a sum of chosen periodic signals of the waveforms in Fig. 1 and of a direct component of a given value (a random noise can be added too). As we are interested not only in improving the Fourier analysers but also in improving the extended Fourier analysers, which process signals consisting of several periodic components of different waveforms (spectra) and (not harmonically related) frequencies, we have to use also test signals like signal 6 , where higher harmonic components exceed the fundamental one significantly. The influence of these higher harmonic components is similar to the influence of the higher frequency periodic components in the extended analyser.

First we consider and compare the properties of the following modifications of such (step-)adaptive Fourier analyser, which finds $\Delta \hat{\omega}$ and updates $\hat{\omega}$ and the analyser's frequency-dependent parameters (see Eqs. (2) and (3)) in every timestep.

RA - the robust AFA, which computes $\mathbf{g}(k)$ using (4) and finds $\Delta \hat{\omega}$ according to

$$
\Delta \hat{\omega}(k)=D \cdot \angle\left(\hat{X}_{1}(k) / \hat{X}_{1}(k-1)\right) / N(k),
$$

where $D$ is a damping factor $(0<D \leq 1)$, which is introduced to improve the precision of $\Delta \hat{\omega}$, performing implicit averaging of the angle $\angle(\cdot)$ over $D^{-1}$ times longer interval than in (8). finds

IRA - the improved robust AFA, which also computes $\mathbf{g}(k)$ using (4) and 


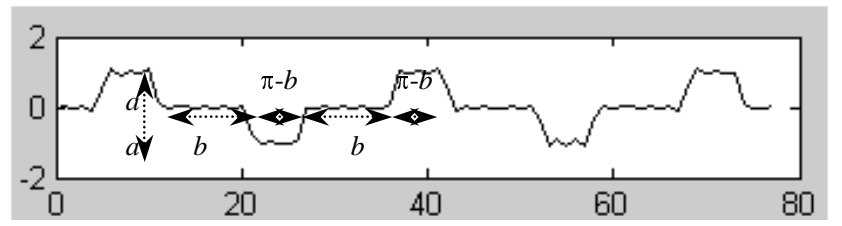

(a) Signal 1

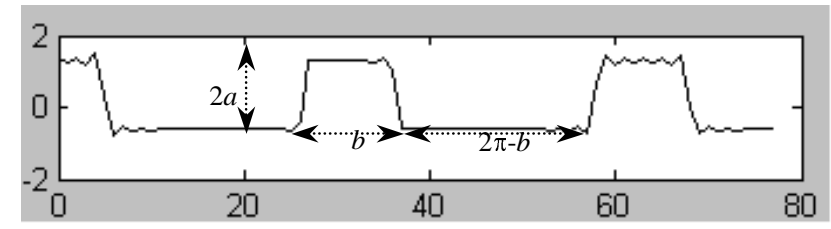

(b) Signal 2

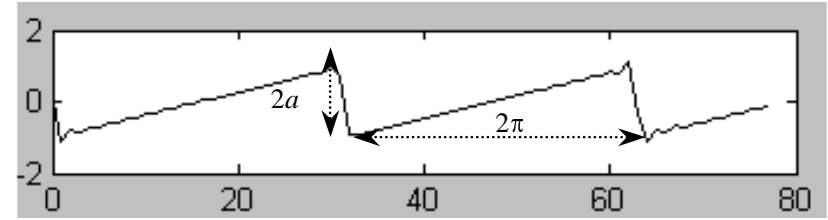

(c) Signal 3

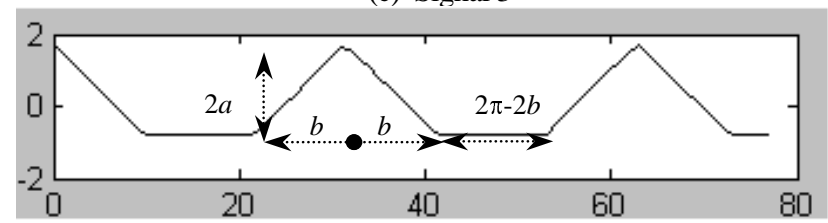

(d) Signal 4

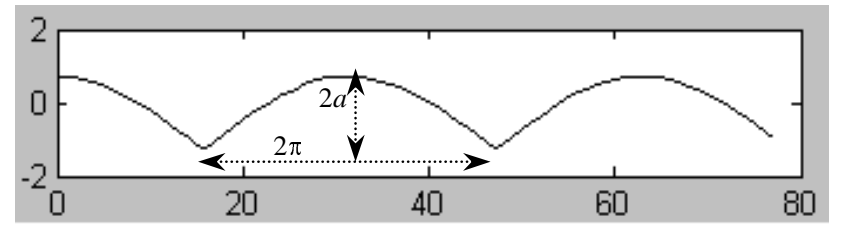

(e) Signal 5

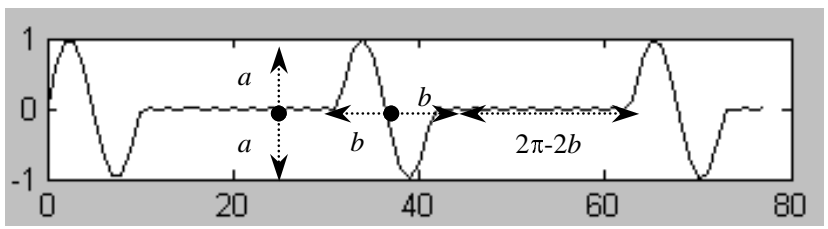

(f) Signal 6

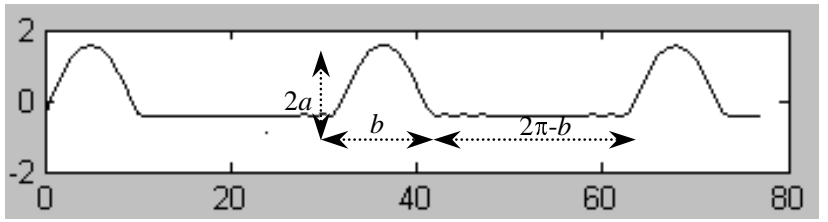

(g) Signal 7

Fig. 2. Plots of periodic band-limited discrete-time signals used to test modifications of the analyser. 


$$
\Delta \hat{\omega}(k)=D \cdot \angle\left(\hat{X}_{1, B}(k) / \hat{X}_{1, B}(k-1)\right) / N(k),
$$

applying vector conditioning (9).

In order to make the AFAs capable to analyse periodic signals where components of higher frequencies dominate, we had to avoid possible convergence of $\hat{\omega}$ to higher harmonic frequencies in case of significant (initial) error $\Delta \omega$. This is done (like in the extended BAFA $\left[^{1,2}\right]$ ) by means of the frequency updating process with the bounds $\omega_{L}$ and $\omega_{U}$ set for $\hat{\omega}_{\text {new }}$ :

$$
\text { if } \omega_{L}<\hat{\omega}+\Delta \hat{\omega}<\omega_{U} \text { then } \hat{\omega}_{\text {new }}=\hat{\omega}+\Delta \hat{\omega} \text { otherwise } \hat{\omega}_{\text {new }}=\left(\hat{\omega}+\omega_{L}\right) / 2 \text {. }
$$

Later we consider characteristics of block-adaptive Fourier analysers: RBA the robust BAFA, in which $\mathbf{g}(k)$ is updated according to (4) and DBA - the dead-beat (observer based) BAFA, in which $\mathbf{g}(k)$ is updated applying (5).

In these analysers frequency estimation is performed only after passing optimal settling-time (which is equal to $N$ sampling intervals not only in the dead-beat BAFA $\left[^{7}\right]$ but also in the robust BAFA) since previous frequency/parameters updating. In both, RBA and DBA the error $\Delta \omega$ is estimated applying (10) and (11) and using different coefficients $C_{1}$ and $C_{2}$ to determine the values of the parameters $B=\operatorname{ceil}\left(C_{1} L\right)$ and $P=\operatorname{ceil}\left(C_{2} L\right)$ (here $\operatorname{ceil}(y)$ is the smallest integer $\geq y>0$ ).

As we have computed the (frequency) convergence-time surfaces $t_{1}\left(C_{1}, C_{2}\right)$, $t_{2}\left(C_{1}, C_{2}\right)$ and $\Delta t\left(C_{1}, C_{2}\right)$ of these analysers and cleared out their local/global minima patterns for several signals $\left[{ }^{9}\right]$, we present in this paper convergence times along some more interesting lines of these surfaces (found by averaging greater sets of simulation results). Using the parameters from these lines is later considered applying the following frequency error's estimation methods:

- the angle enlarging method $\mathrm{AE}$ uses $C_{1}=L^{-1}$ (i.e. $B=1$ in (11)) and $C_{2}=C$;

- the vector averaging method $\mathrm{VA}$ uses $C_{1}=C$ and $C_{2}=L^{-1}$ (i.e. $P=1$ in (10));

- the combined methods:

method VA\&AE uses $C_{1}=C_{2}=C$ (in the DBA the minimum is on or near this line);

method $\mathrm{VA}<\mathrm{AE}$ uses $4 C_{1}=C_{2}=C$ (in the RBA the minimum can be on or near this line).

\section{SIMULATION RESULTS}

\subsection{Experiments with (step-)adaptive Fourier analysers}

The experiments with the RA, some results of which are presented in Fig. 3, showed that for the signals $1-5$ (with $1 / f$-type or faster decreasing spectrum) transients of the frequency $\hat{\omega}$ are time-optimal (i.e. have maximal average convergence speed found as a change of $20 \log _{10}\left|\delta_{\omega}\right|$ in $\mathrm{dB}$ per $T$ ) in the neighbour- 


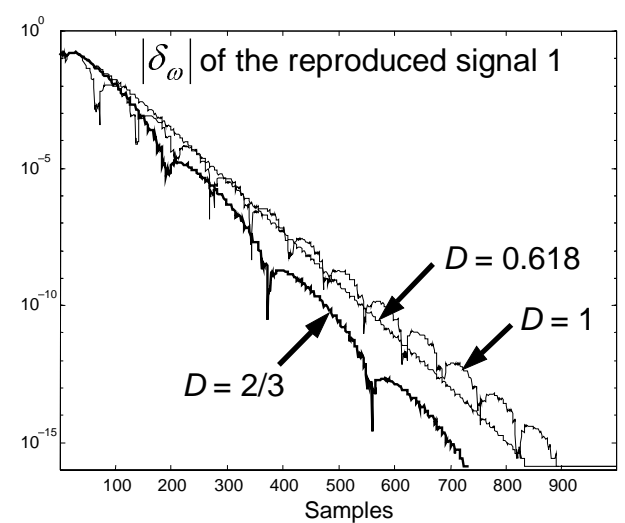

(a)

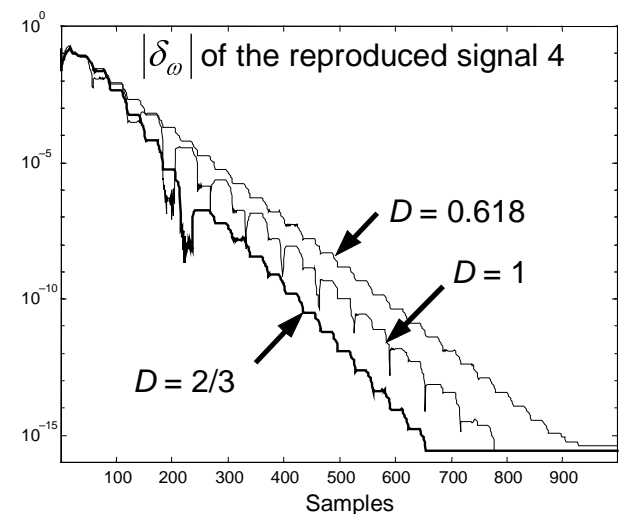

(c)

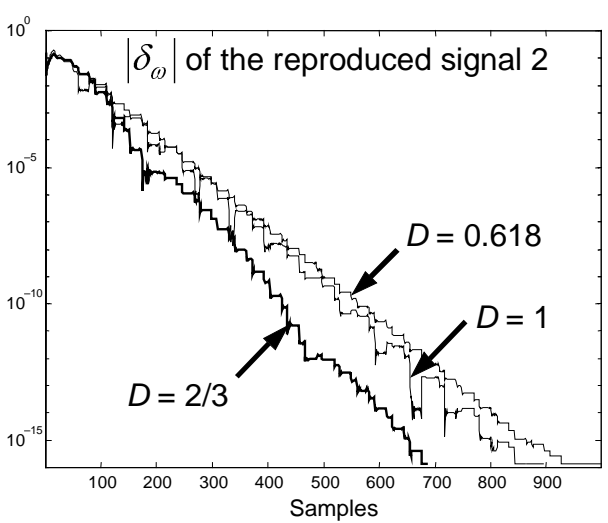

(b)

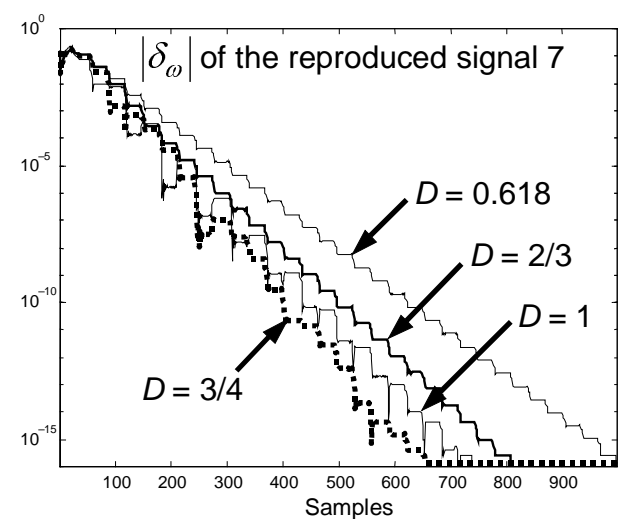

(d)

Fig. 3. Convergence of the module of the relative frequency error $\left|\delta_{\omega}\right|$ in the robust adaptive Fourier analyser; $\left|\delta_{\omega}\right|$ is given in a logarithmic scale; the initial error is $\delta_{\omega}=0.15$.

hood of $D=2 / 3$ or even $D=3 / 4$ (signals 3 and 7). Frequency transients in the original robust AFA $\left.{ }^{6}\right]$, which uses $D=1$, were more oscillating (with significant frequency overshooting), but the golden section ratio $D=0.618$ gave us already aperiodic transients of $\hat{\omega}$.

Let us consider the frequency transient processes in more detail in case of signal 6 , several higher harmonic components of which exceed the fundamental one significantly and make it impossible to analyse this signal in AFA $\left[{ }^{6}\right]$ and BAFA $\left[{ }^{7}\right]$. Figure 4 shows that using the introduced parameter $D$, one can make the RA capable to process such periodic signals too. Of course, the convergence speed of the error $\left|\delta_{\omega}\right|$ (in $\mathrm{dB}$ per $T$ ) in the time-optimal frequency transient process (achieved using $D \approx 0.45$, see Fig. $4 \mathrm{a}$ ) is then more than 3.5 times slower than that for the other signals (see Fig. 3). Increasing of $D$ makes the convergence slower after achieving $\left|\delta_{\omega}\right| \approx 0.01$ and the error's tendency to diverge becomes 


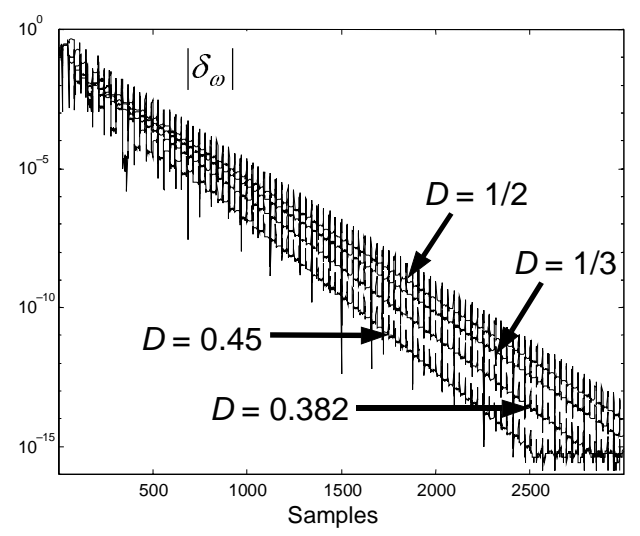

(a)

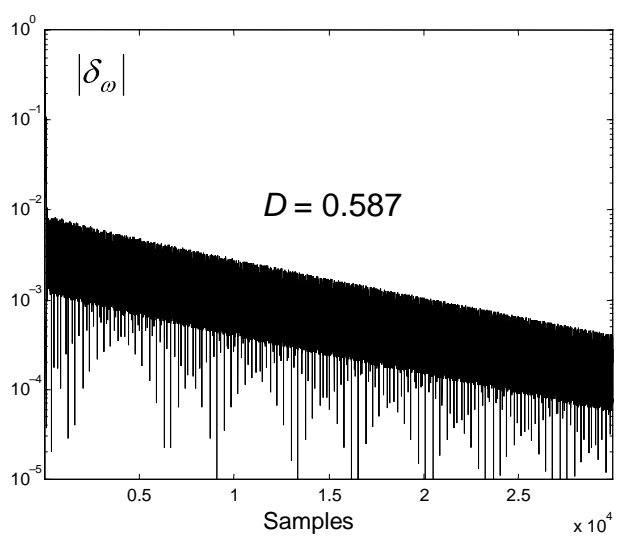

(c)

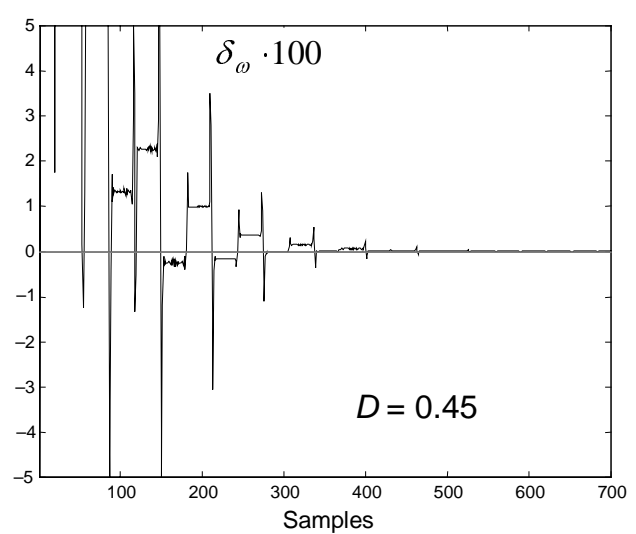

(e)

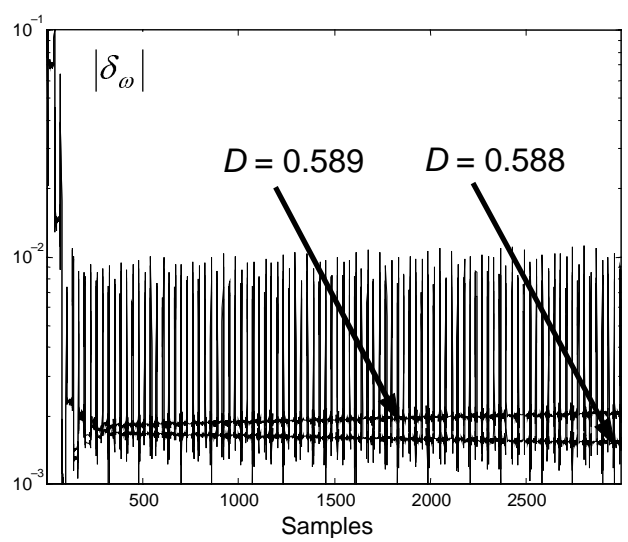

(b)

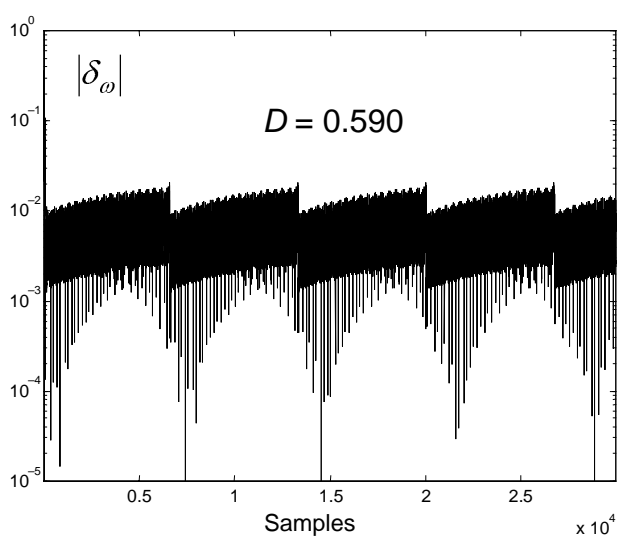

(d)

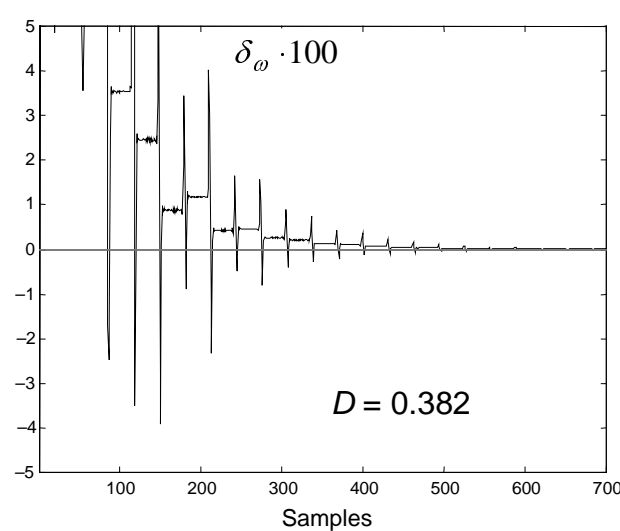

(f)

Fig. 4. Convergence of the relative frequency error $\delta_{\omega}$ of the reproduced signal 6 in the robust adaptive Fourier analyser; $\left|\delta_{\omega}\right|$ is given in a logarithmic scale; the initial error is $\delta_{\omega}=0.15$. 
apparent above the level $D=0.588$. Figure 4 demonstrates also the behaviour of $\delta_{\omega}$ in time-optimal and close-to-aperiodic $(D=0.382)$ transient processes.

The IRA applies vector conditioning to improve frequency error estimation and to speed up frequency convergence in case when the analysed input signal contains dominating higher frequency components. All the results presented in the following have been obtained applying vector conditioning over $T$, i.e. $B=N$ in (11), which ensured time-optimal convergence of the frequency $\hat{\omega}$ to $\omega$.

Our experiments (see Figs. 5 and 6) showed that in the IRA time-optimal frequency convergence can be achieved using values of $D$ chosen from the neighbourhood of 0.382 (the square of the golden section ratio), i.e. $1 / 3<D<2 / 5$, and $D=1 / 3$ ensures for most signals aperiodic transients of $\hat{\omega}$.

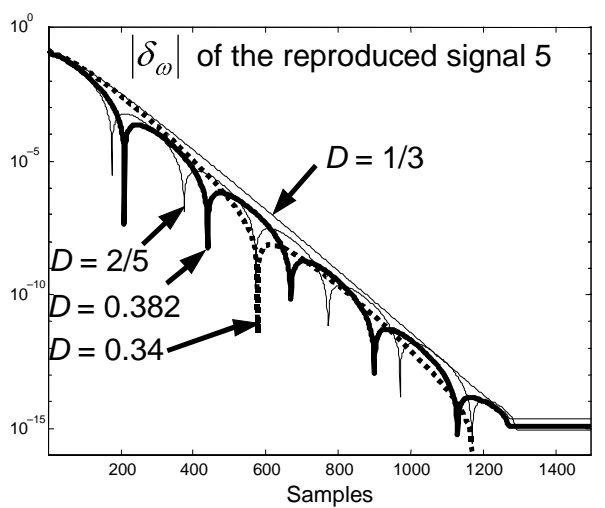

(a)

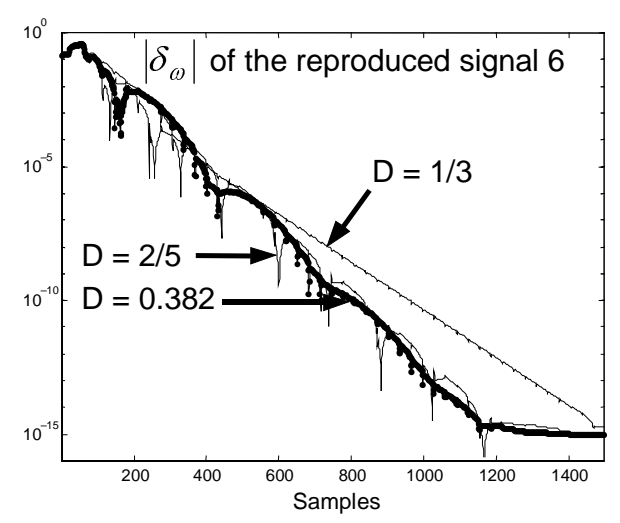

(c)

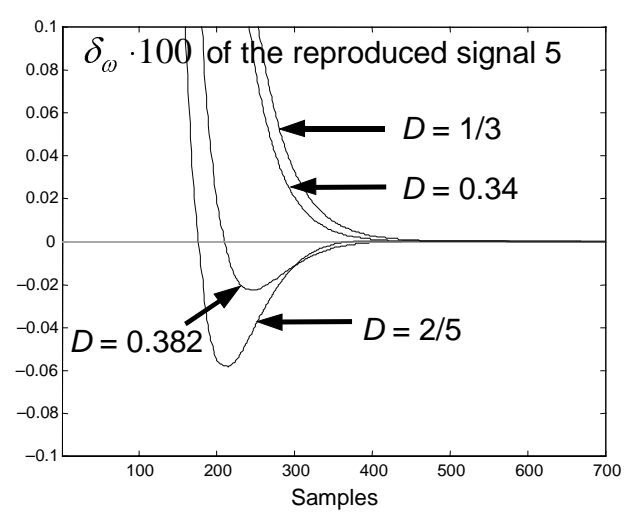

(b)

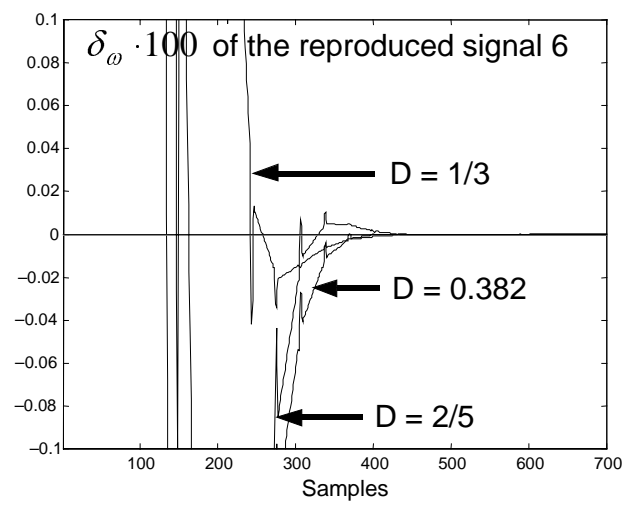

(d)

Fig. 5. Convergence of the relative frequency error $\delta_{\omega}$ in the improved robust adaptive Fourier analyser (IRA) where $B=N$ is used in (11); the curves $\delta_{\omega}$ of the signals $1-5$ and 7 are very close to each other; $\left|\delta_{\omega}\right|$ is given in a logarithmic scale; the initial error is $\delta_{\omega}=0.15$. 


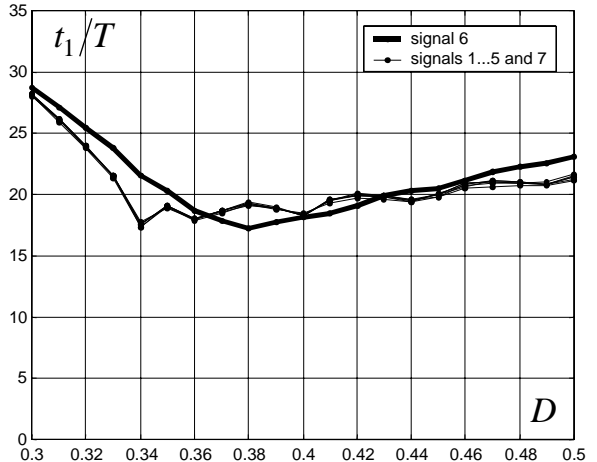

(a)

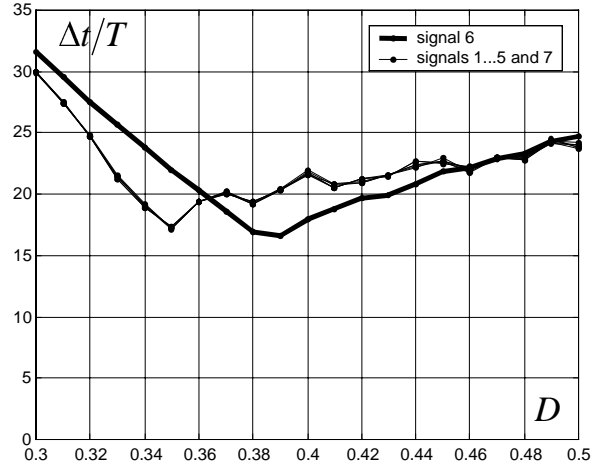

(b)

Fig. 6. The normalized average (frequency) convergence times $t_{1} / T$ and $\Delta t / T$ vs the damping factor $D$ for the improved robust adaptive Fourier analyser (IRA) where $B=N$ in (11); the times have been averaged over 60 experiments: 30 different randomly chosen initial phases of the input signal were used and signal analysis was performed starting from initial frequencies $\hat{\omega}$ with the errors $\delta_{\omega}=+0.15$ and $\delta_{\omega}=-0.15$.

In case of signals $1-5$ and 7 , the optimal value $D=0.35$ leads to twice slower and in case of signal 6 the optimal $D=0.382$ leads to nearly twice faster frequency convergence as compared to the time-optimal frequency convergence in the RA. In case of signal 6, nearly time-optimal transients of $\hat{\omega}$ oscillate in the beginning but become later aperiodic; purely aperiodic transients are achieved below the level $D=1 / 3$.

Figure 6 presents one main result: the curves of normalized average convergence times $t_{1} / T$ and $\Delta t / T$ vs $D$ for the test signals 1-7 processed in the IRA. As these times depend on an initial phase of the input signal, the averaging was performed over 60 experiments, in which 30 randomly chosen initial phases and the initial errors $\delta_{\omega}= \pm 0.15$ were used.

In the robust analyser IRA the convergence of $\hat{\omega}$ to values of $\omega$, which lie in small regions just below the values $\pi / n$ ( $n$ is an integer) is slower or even limited and Fig. 7 demonstrates that in such regions the convergence process becomes oscillatory when a certain (slightly varying) frequency error level is achieved. The error $\delta_{\omega}$ oscillates with the signal's frequency $\omega \approx \hat{\omega}$, at the same time the error signal $\varepsilon=x-\hat{x}$ oscillates with the angular frequency $\pi$. Analogous phenomenon has been observed in the dead-beat AFA too, but in much smaller regions $\left.{ }^{10}\right]$. Figure 8 shows that if the bandwidth of the input signal is smaller (smaller than the bandwidth of the analyser, $M<L$ ), then the critical error level (where oscillations appear) becomes lower. Thus pre-filtering of the input signal permits only to suppress (to some extent) this phenomenon. The latter becomes more disturbing when we try to use lower sampling rates and to reduce in this way the amount of computations per time unit.

In the end we have to state that the dead-beat (observer based) AFA, which uses (4) instead of (3) to update its parameters, does not suit for adaptation in 


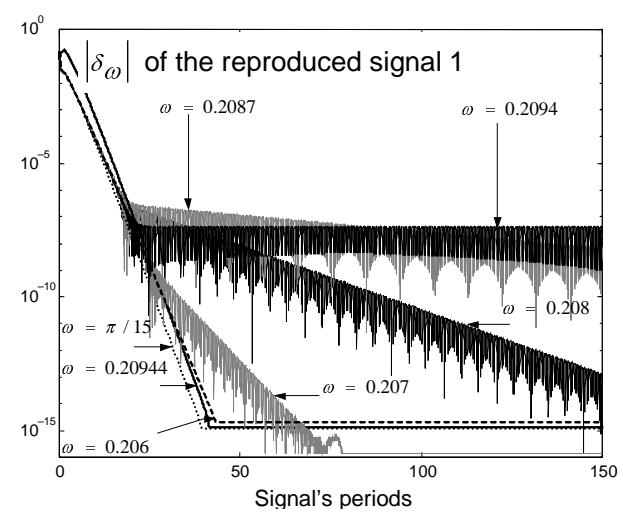

(a)

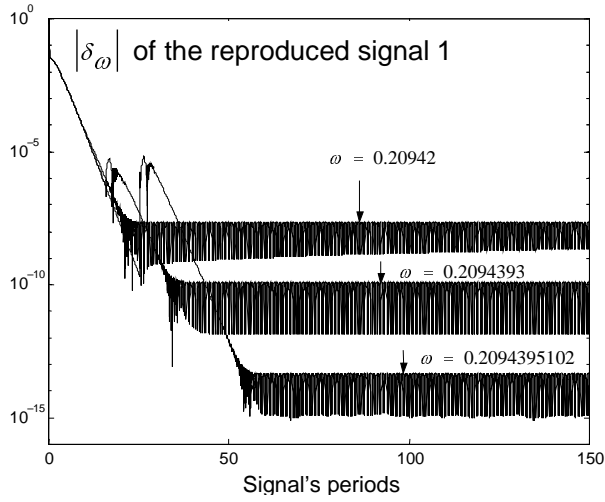

(b)

Fig. 7. Convergence of the frequency error $\left|\delta_{\omega}\right|$ in the improved robust adaptive Fourier analyser ( $B=N$ is used in (11) and $D=1 / 3$ ) when $\omega$ is in the neighbourhood of $\pi / 15 ;\left|\delta_{\omega}\right|$ is given in a logarithmic scale, the initial error is $\delta_{\omega}=0.15$.

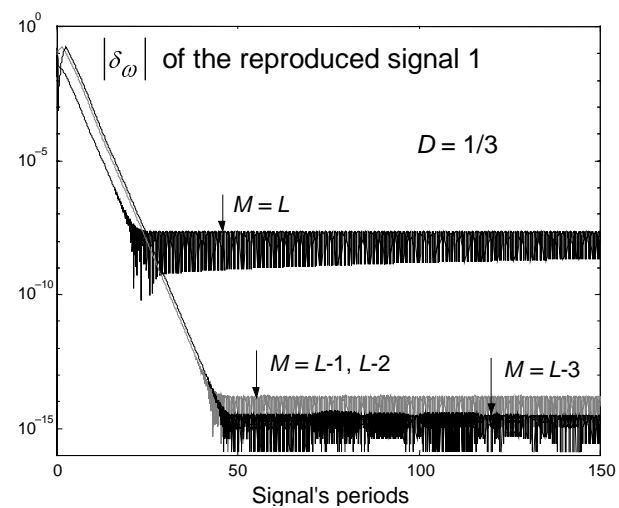

(a)

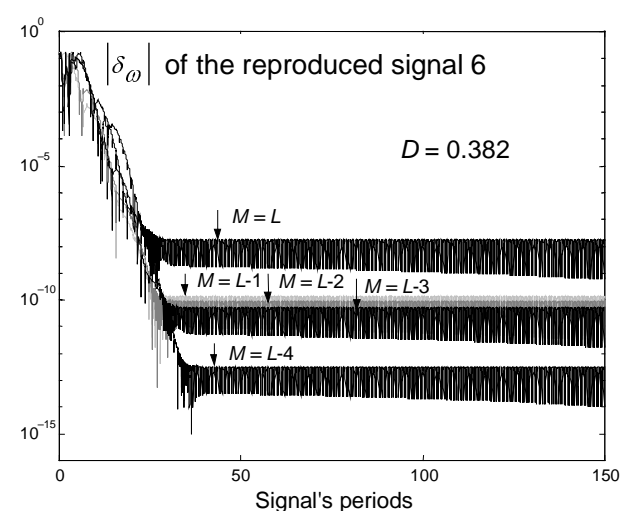

(b)

Fig. 8. Convergence of the frequency error $\left|\delta_{\omega}\right|$ in the improved robust adaptive Fourier analyser $(B=N$ is used in (11)) when the input signal's frequency $\omega=0.20942$ is in the neighbourhood of $\pi / 15$ and $M$, the number of formed Fourier components of the input signal, is equal or smaller than $L$, the number of Fourier components computed/reproduced in the analyser; $\left|\delta_{\omega}\right|$ is given in a logarithmic scale, the initial error is $\delta_{\omega}=0.15$.

every time-step. It is computationally less efficient and it works much worse in case of significant frequency errors and with signals containing more disturbing higher harmonic components. Its frequency convergence is not worse than in the robust AFA only in case of very small frequency errors. Vector conditioning makes the dead-beat AFA's behaviour better, as it suppresses disturbing influence of its transients and possible oscillations, which have not been noticed in the robust AFA (see Fig. 9). 


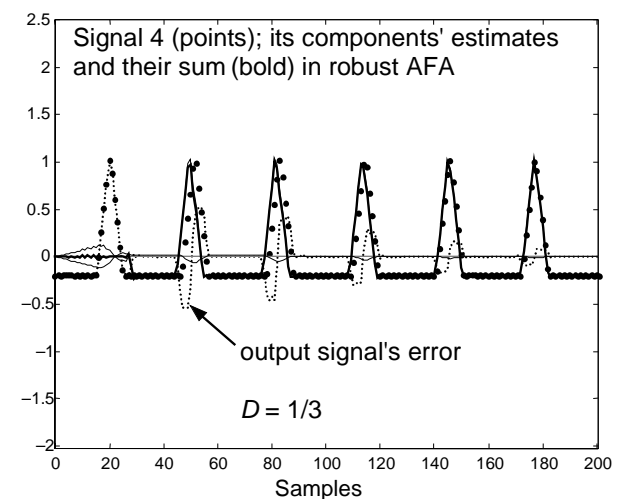

(a)

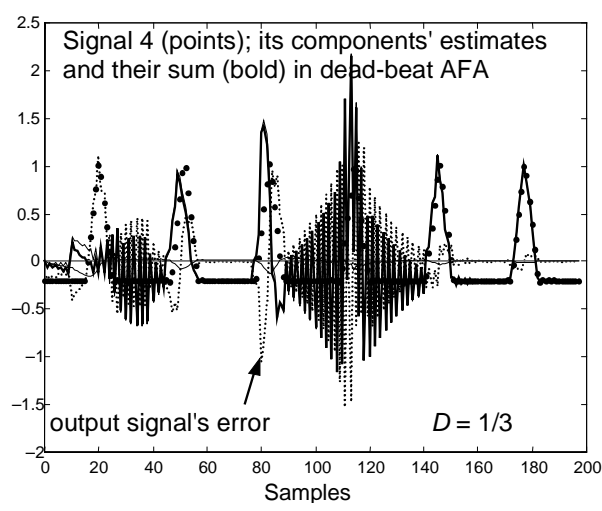

(b)

Fig. 9. Transients in the (step-)adaptive Fourier analysers, which apply vector conditioning over the input signal's period; the initial frequency error is $\delta_{\omega}=0.15$.

\subsection{Experiments with block-adaptive Fourier analysers BAFA}

In this section we consider the simulation results obtained with the described modifications of BAFA: DBA and RBA. Some additional results on these analysers can be found in $\left[{ }^{9}\right]$.

Figure 10 presents dependence of the normalized convergence times $t_{1} / T$ and $\Delta t / T$ on the parameter $C$ for two signals analysed in the DBA. In the plots in these figures the convergence time curves are given for three block-adaptation methods (frequency error estimators) of interest. These curves have several local minima, which correspond to the signal's period and significant abrupt changes in the signal's waveform [ $\left.{ }^{9}\right]$.

One can see that in the DBA the VA\&AE method gives, in spite of two times longer error estimation cycle, up to two times shorter convergence times compared to the AE or VA method. It is more efficient 1) in case of small frequency errors (the plots of $\Delta t / T$ in Fig. 10), 2) in case of signal 1 and also signal 2 with $b=\pi$, which both have even spectrum lines equal to zero (see $\left[{ }^{9}\right]$ ), and 3 ) in case of the signal 6 with dominating higher harmonic components (Figs. 10c, d, e and f).

It appears that the VA\&AE method is, in general, most efficient if $B \approx P \approx \operatorname{ceil}(2 \pi / \hat{\omega})$, but in case of signals, even spectrum lines of which are zero, $B \approx P \approx \operatorname{ceil}(\pi / \hat{\omega})$ gives better results.

In the RBA transient processes do not decay in $N$ steps as in the DBA and thus its frequency convergence speed (see Fig. 11) is about $2 / 3$ of that of the DBA (see Fig. 10), but greater than in the IRA (Fig. 6) for all the used signals. In this analyser the $\mathrm{VA}<\mathrm{AE}$ method with $4 B \approx P \approx \operatorname{ceil}(2 \pi / \hat{\omega})$ and the $\mathrm{AE}$ method with $P \approx \operatorname{ceil}(2 \pi / \hat{\omega})$ are optimal for the signals $1-4$ (the $\mathrm{VA}<\mathrm{AE}$ method with $B \approx 4 P \approx \operatorname{ceil}(2 \pi / \hat{\omega})$ and the VA method with $P \approx \operatorname{ceil}(2 \pi / \hat{\omega})$ are slightly worse). However, the VA\&AE method appears to be the best for processing the signal 6 with dominating higher harmonic components and it is time-optimal in case of $B \approx P \approx \operatorname{ceil}(2 \pi / \hat{\omega})$ as in the DBA. 


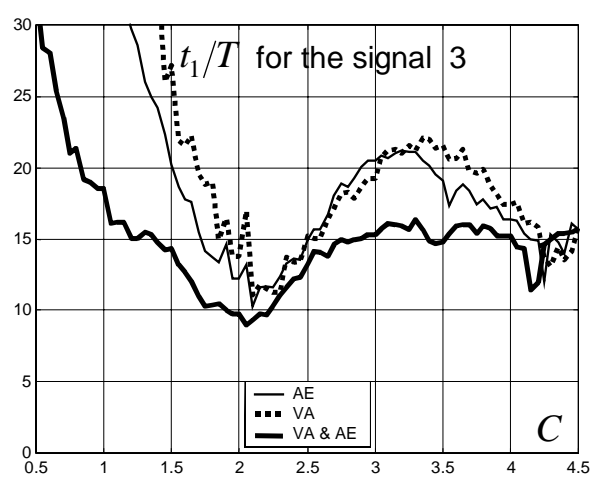

(a)

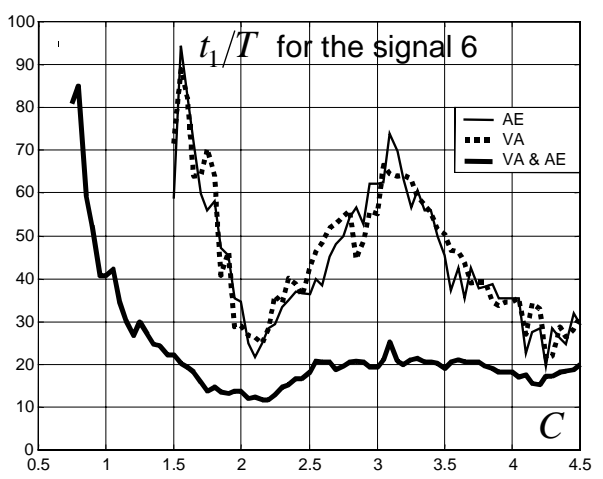

(c)

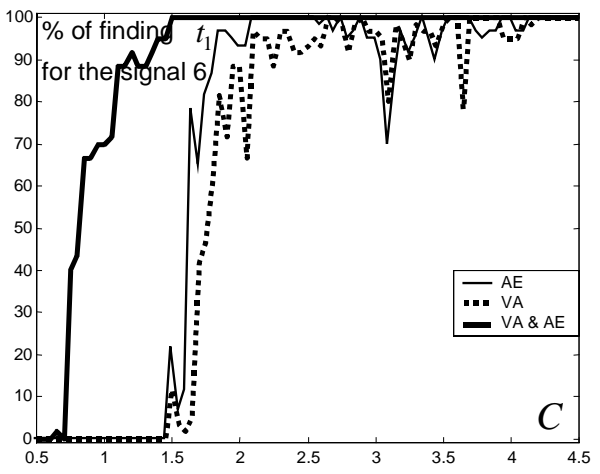

(e)

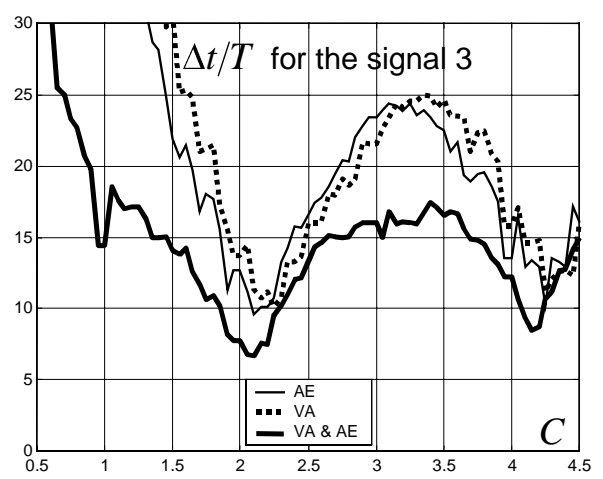

(b)

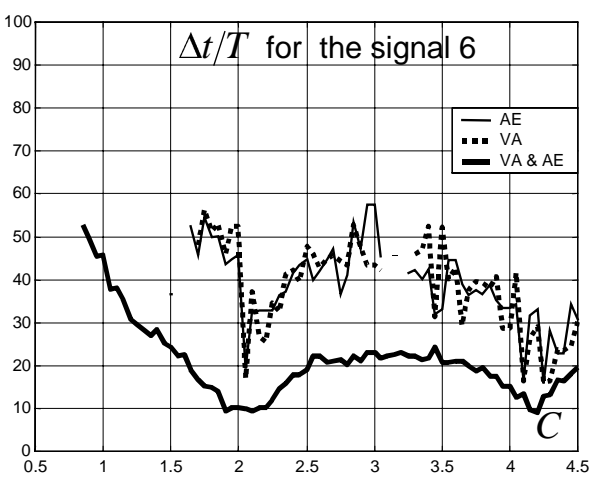

(d)

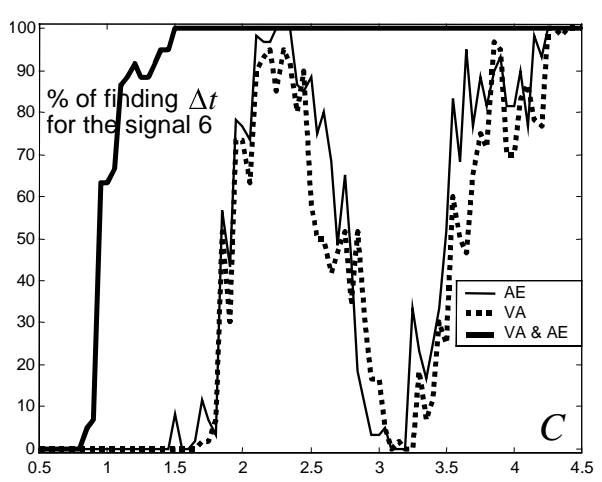

(f)

Fig. 10. The frequency convergence times $t_{1} / T$ and $\Delta t / T$ vs $C$ for the dead-beat BAFA (DBA); the times have been averaged over 60 experiments: 30 randomly chosen initial phases of the input signal and the initial frequencies $\hat{\omega}$ with the errors $\delta_{\omega}= \pm 0.15$ were used; processing the signal 6 the AE- and VA-method could not achieve the error level $\left|\delta_{\omega}\right|=10^{-14}$ in all the 60 experiments for several values of $C$ (broken curves in the plot (d), see also (f)), but the VA \& AE method with $C \geq 1.5$ achieved this level in all the experiments. 


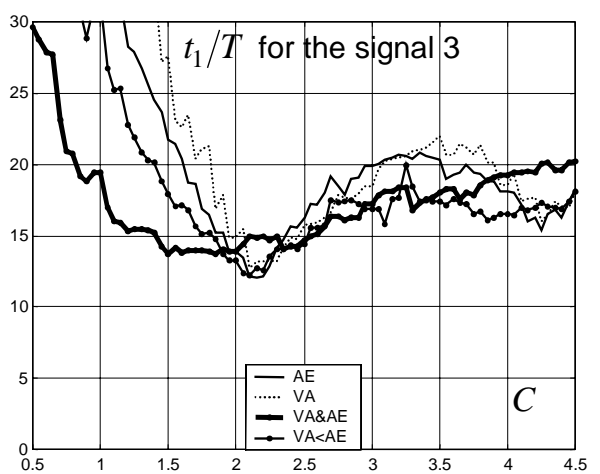

(a)

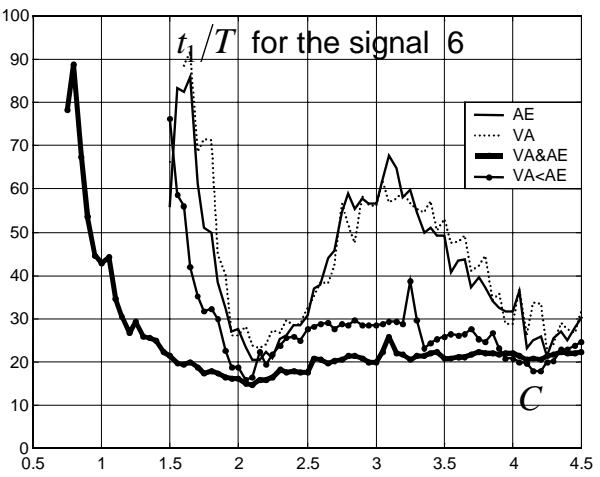

(c)

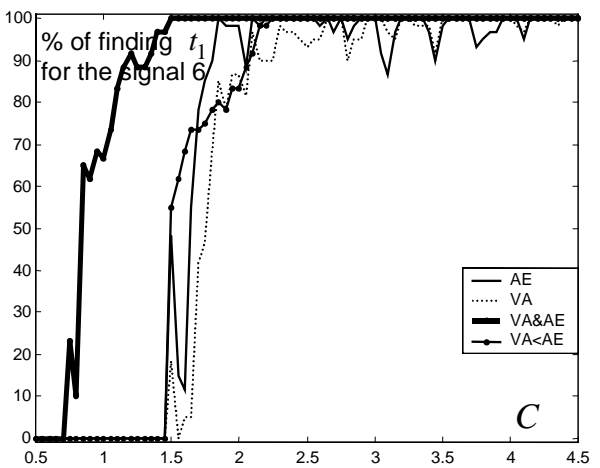

(e)

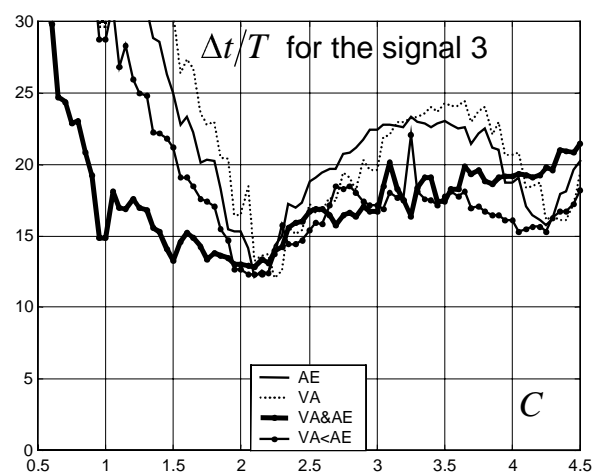

(b)

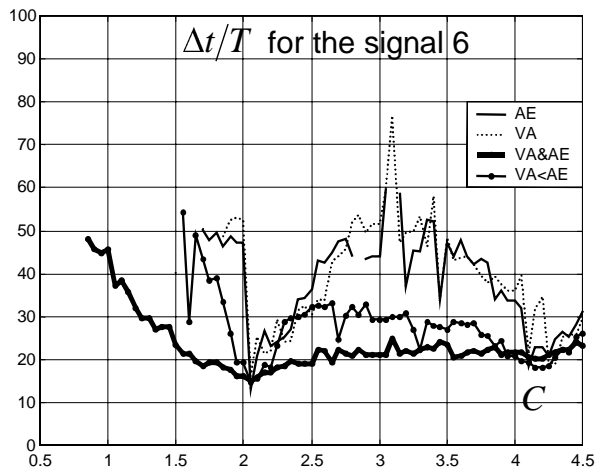

(d)

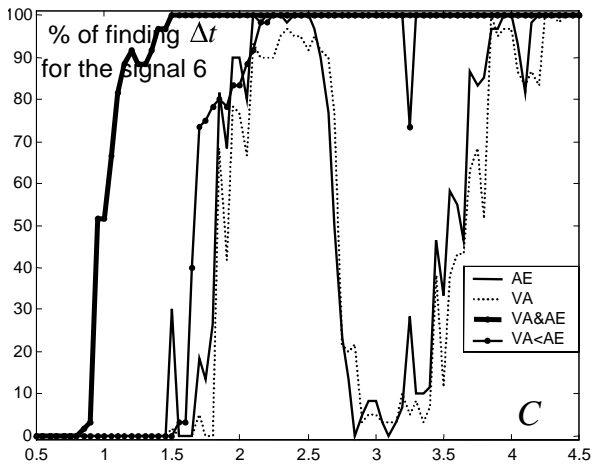

(f)

Fig. 11. The normalized frequency convergence times $t_{1} / T$ and $\Delta t / T$ vs $C$ for the robust BAFA (RBA). These times were found as those in Fig. 10 computing with 3000 time-steps; processing the signal 6 (with dominating higher harmonic components) the VA \& AE method with $C \geq 1.5$ works perfectly (achieves the frequency error level $\left|\delta_{\omega}\right|=10^{-14}$ in all the 60 experiments), the $\mathrm{VA}<\mathrm{AE}$ becomes reliable when $C \geq 2.2$, the $\mathrm{AE}$ and $\mathrm{VA}$ are worse.

Figure 12 permits to compare, how the transient times $t_{1} / T$ and $t_{2} / T$ depend on the input signal's frequency $\omega$ in the analysers DBA and RBA. In the RBA 
(a) Signal 1 in DBA

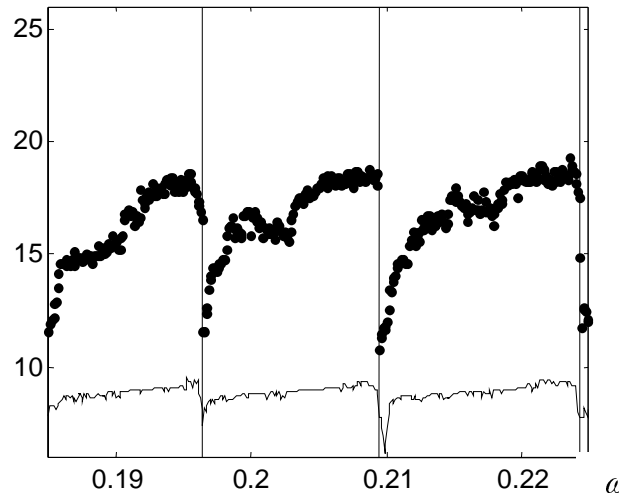

(c) Signal 3 in DBA

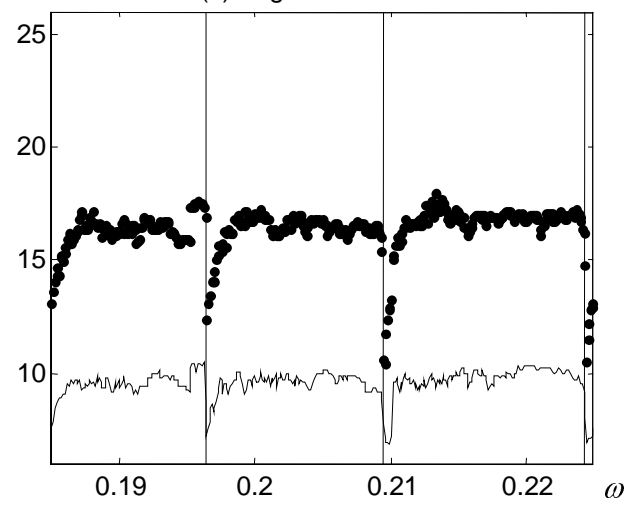

(e) Signal 3 in RBA

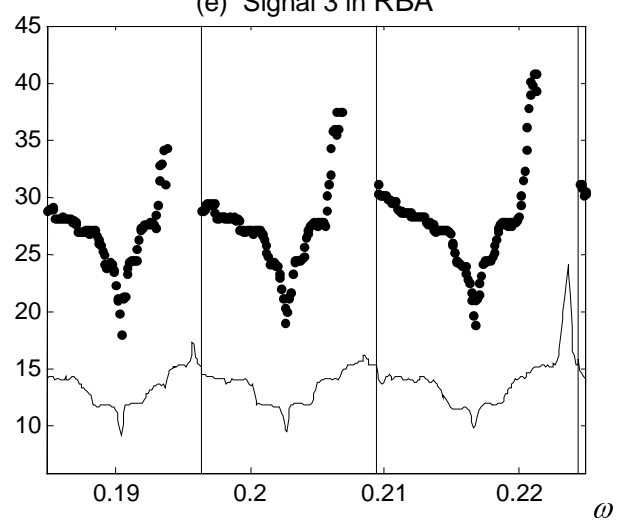

(b) Signal 2 in DBA

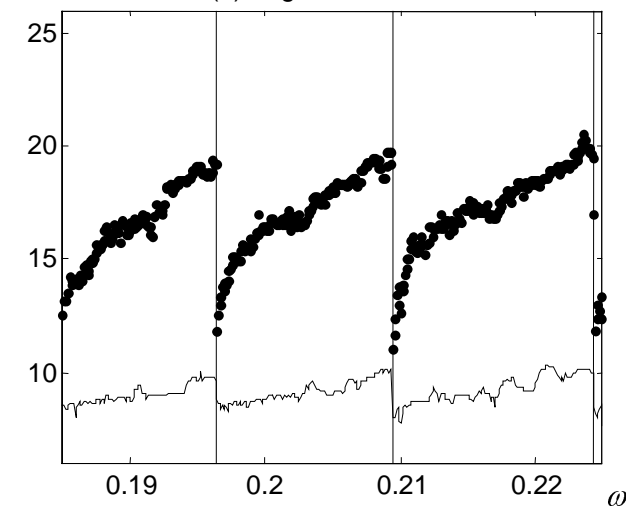

(d) Signal 6 in DBA

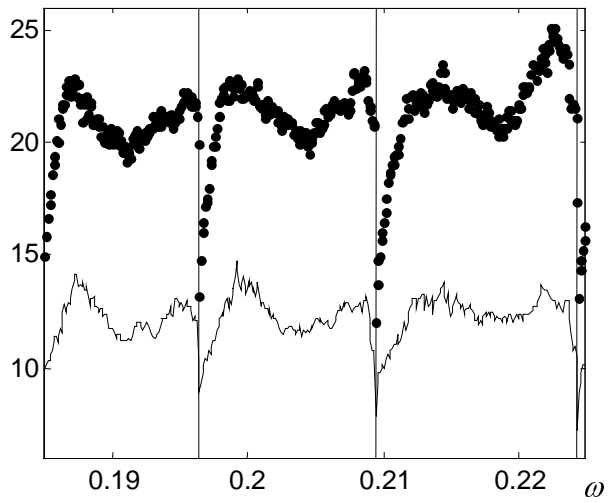

(f) Signal 6 in RBA

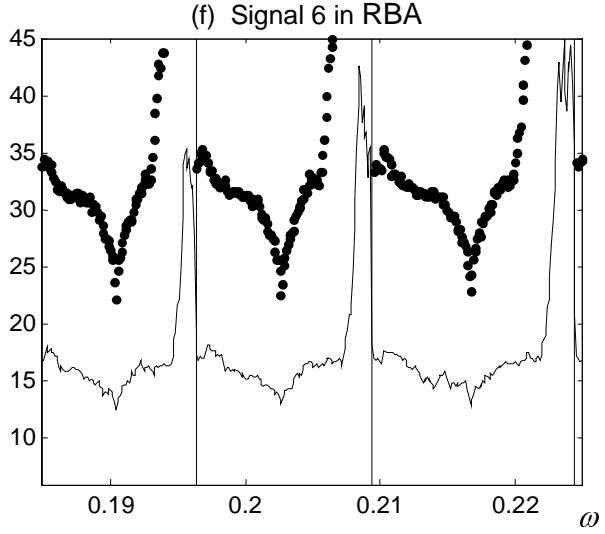

Fig. 12. Averaged transient times $t_{1} / T$ (continuous curves) and $t_{2} / T$ (dotted curves) vs $\omega$ for the analysers DBA and RBA with $C_{1}=C_{2}=2.1$; these times have been averaged over 20 experiments where an input signal of a randomly chosen initial phase was used; the verticals correspond to the frequencies $\omega=\pi / n$ (n-integer), the initial error $\delta_{\omega}=+0.15$. 


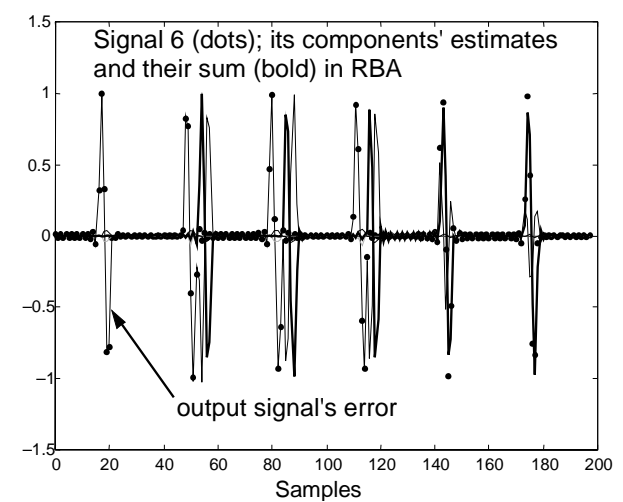

(a)

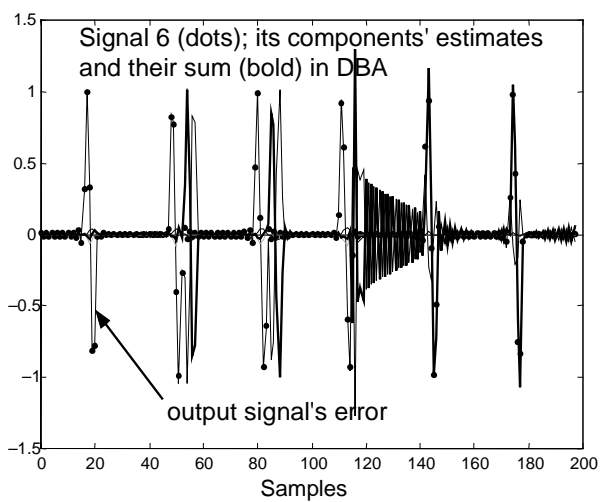

(b)

Fig. 13. Transients in the block-adaptive Fourier analysers, which apply the VA\&AE method with optimal $C=2.15$; the initial frequency error is $\delta_{\omega}=-0.15$.

this dependence is nearly the same for the signals $1-5$ and 7 , and for all the signals there are clear sharp minima on the frequencies $\pi /(n+0.5)$. In the DBA this dependence is more influenced by the signal's waveform and minima of transient times lie just above the frequencies $\pi / n$. The regions of bad convergence, which lie below the lines $\omega=\pi / n$ and were considered in Section 4.1, are very small for the dead-beat analyser, but quite remarkable for the robust analyser, especially in case of the signal 6 (due to its dominating components of higher frequencies). Thus Fig. 12 generalizes the results shown in Fig. 7 and shows that a properly chosen sampling rate can reduce the convergence time up to 30 per cent (the peaks below the values $\omega=\pi / n$ can be suppressed by means of pre-filtering of the input signal).

Last but not least, in the RBA, we have not observed oscillations of the angular frequency $\pi$ met in transients in the DBA (see Fig. 13).

\section{CONCLUSIONS}

The obtained simulation results permit to state the following.

1. Introducing the damping factor $D<1$ into the robust AFA (its frequency error estimator) one can a) improve average frequency convergence speed of the analyser up to 20 per cent and b) make the analyser capable to process signals with dominating higher harmonic components.

2. To perform efficient analysis of signals with dominating higher harmonic components one has to suppress disturbances in the frequency error estimator of robust or dead-beat AFA (and also BAFA) applying conditioning/averaging of the 1st Fourier coefficient vector (over the signal's period $T$ ) and performing thus more computing operations per sample.

3. The frequency convergence time characteristics $\left(t_{1} / T\right.$ and $\Delta t / T$ vs the error estimator's parameters), computed for different analysers (signals), can be used to 
find proper or even optimal analyser parameters for solving certain signal processing tasks.

4. The undesired significant oscillations (which appear in transients in deadbeat analysers and become often very disturbing in the extended BAFA $\left[{ }^{2}\right]$ ) have not been observed in robust analysers. Thus we can suggest that it would be better to extend a robust analyser for processing composite signals, which consist of periodic components of different (non-harmonic) frequencies and waveforms. Presumably the robustness makes the analyser more reliable.

\section{ACKNOWLEDGEMENT}

The authors wish to thank the reviewers for useful comments and the Estonian Science Foundation for supporting this work (grant No. 5902).

\section{REFERENCES}

1. Ronk, A. On frequency adaptation in an extended block-adaptive Fourier analyser. Proc. Estonian Acad. Sci. Eng., 2001, 7, 309-330.

2. Ronk, A. Analysis and reproduction of a signal's periodic components by means of an extended block-adaptive Fourier analyser. IEEE Trans. Instrum. Meas., 2003, 52, 13-19.

3. Hostetter, G. H. Fourier analysis using spectral observers. Proc. IEEE, 1980, 68, 284-285.

4. Hostetter, G. H. Recursive discrete Fourier transformation. IEEE Trans. Acoust., Speech Signal Process., 1980, 28, 184-190.

5. Péceli, G. A common structure for recursive discrete transforms. IEEE Trans. Circuits Syst., 1986, 33, 1035-1036.

6. Nagy, F. Measurement of signal parameters using nonlinear observer theory. IEEE Trans. Instrum. Meas., 1992, 41, 152-155.

7. Simon, Gy. and Péceli, G. Convergence properties of an adaptive Fourier analyzer. IEEE Trans. Circuits Syst.-II, Analog Digit. Signal Process., 1999, 46, 223-227.

8. Ronk, A. A modified frequency error estimator for the block adaptive Fourier analyzer. In Proc. Baltic Electronics Conference (BEC'2000). Tallinn, 2000, 103-106.

9. Voolaine, Ü. and Ronk, A. Comparison of some frequency adaptation methods in different block-adaptive Fourier analysers. In Scientific Proc. of Riga Technical University, Series 7: Telecommunications and Electronics, 2003, 3, 63-68.

10. Ronk, A. Frequency pattern fitting in an extended block-adaptive Fourier analyser - I: indispensable restrictions. In Proc. Baltic Electronics Conference (BEC'2002). Tallinn, 2002, 153-156.

\section{Siirdeprotsessid adaptiivsetes Fourier' analüsaatorites}

\section{Ants Ronk ja Ülle Voolaine}

On käsitletud mõningaid adaptiivse Fourier' analüsaatori modifikatsioone ning esitatud rida simuleerimistulemusi, mis võimaldavad võrrelda nende modifikatsioonide koonduvusomadusi ja leida teatud rakenduste jaoks nii analüsaatori sobivaim modifikatsioon kui ka selle parameetrite optimaalsed väärtused. 